Las Torres de Lucca. Revista internacional de filosofía política ISSN-e: 2255-3827

\title{
Los minipúblicos deliberativos y la concepción populista de la representación como "encarnación" del pueblo1
}

Cristina Lafont

Recibido: 10-5-2021 / Aceptado: 25-08-2021/ Publicado: 30-01-2022

Resumen. En este trabajo analizo las propuestas de insertar minipúblicos deliberativos en el proceso político para superar las numerosas "brechas" de representación que aquejan actualmente a los sistemas de partidos tradicionales. Sostengo que la noción de representación que subyace a muchas de estas propuestas tiene algunas similitudes importantes con la noción de representación como "encarnación" del pueblo propia del populismo. A partir de un análisis comparativo entre las variedades populistas y lotocráticas de la representación como "encarnación" del pueblo, destaco dos características que comparten y que son perjudiciales para la democracia. Sobre la base de este análisis, defiendo una forma de entender la relación entre los participantes de los minipúblicos y la ciudadanía en general que evita los rasgos problemáticos de la concepción populista de la representación como encarnación. Para finalizar, expongo cómo las propuestas de usos de los minipúblicos basados en la "confianza" ciega es problemática y abogo, en cambio, por usos participativos de los minipúblicos que realmente empoderarían a la ciudadanía.

Palabras clave: democracia; minipúblicos; lotocracia; participación ciudadana; representación; populismo.

\section{[en] Deliberative Minipublics and the Populist Conception of Representation as Embodiment}

Abstract. In this essay, I analyse different proposals for inserting deliberative minipublics into the political process in order to overcome the many representational "gaps" that afflict traditional party systems today. I argue that the notion of representation that underlies many of these proposals has some strong similarities to the notion of representation as "embodiment" that is characteristic of populism. From a comparative analysis of the populist and lottocratic varieties of "embodied representation," I highlight two features they share, which are also inimical to democracy. On the basis of this analysis, I defend a way of understanding the relationship between minipublics' participants and citizenry at large that avoids the problematic features of the conception of representation as embodiment. To conclude, I show how defences of "trust-based" uses of minipublics can be problematic and, instead, argue for participatory uses of minipublics that would genuinely empower the citizenry.

Keywords: democracy; minipublics; lottocracy; public participation; representation; populism.

Cómo citar: Cómo citar: Lafont, C. (2022). Los minipúblicos deliberativos y la concepción populista de la representación como “encarnación” del pueblo. Las Torres de Lucca. Revista internacional de filosofia política, 11(1), 13-21. https://dx.doi.org/10.5209/ ltdl.79872

En medio del creciente descontento con las democracias representativas, nuevas formas de participación política directa de los ciudadanos son cada vez más populares. En este contexto, han proliferado las propuestas para insertar minipúblicos deliberativos en el proceso político, tales como asambleas ciudadanas, jurados de ciudadanos o encuestas deliberativas. De hecho, muchos teóricos de la democracia ven estos mecanismos como la mejor esperanza para superar las numerosas "brechas" de representación que aquejan a los sistemas de partidos tradicionales - brechas que han producido niveles alarmantes de apatía y desencanto de los ciudadanos con la democracia-. Sin embargo, a pesar del interés que han suscitado estas nuevas formas de participación ciudadana, se ha analizado poco la naturaleza específica de la representación política que implican. ${ }^{2}$ ¿En qué

Nota de las traductoras:

Traducción: Luciana Wisky, Universidad de Buenos Aires, Argentina.

Correo electrónico: lucianawisky@gmail.com

ORCID: https://orcid.org/0000-0002-2292-0639

Revisión: Moira Pérez, Universidad de Buenos Aires, Consejo Nacional de Investigaciones Científicas y Técnicas, Argentina.

Correo electrónico: perez.moira@gmail.com

ORCID: https://orcid.org/0000-0002-3364-7949

2 De hecho, una de las razones más destacadas de esta laguna es que estas instituciones innovadoras suelen considerarse como lugares de participación directa de los ciudadanos y, por lo tanto, no se reconoce su función representativa. Como explica convincentemente Warren: "Debido en 
sentido se supone que los ciudadanos que participan en los minipúblicos son representantes del resto de la ciudadanía? ¿Pueden los ciudadanos actuar al mismo tiempo como ciudadanos de pleno derecho y como representantes de quienes no participan?

En este ensayo sostengo que la noción de representación que subyace a muchas de las propuestas para insertar minipúblicos en el proceso político tiene algunas similitudes importantes con la noción de representación como "encarnación"3* del pueblo propia del populismo. Luego de analizar algunas diferencias entre las variedades populistas y lotocráticas de la representación como "encarnación," destaco dos características que comparten y que son altamente perjudiciales para la democracia: el mayoritarismo excluyente y el supuesto de homogeneidad política de la ciudadanía. En contra de este punto de vista, defiendo una forma de entender la relación entre los participantes de los minipúblicos y la ciudadanía en general que evita los rasgos problemáticos de la concepción de la representación como encarnación. Sobre la base de esta comprensión alternativa, extraigo algunas consecuencias prácticas respecto a propuestas actuales para usos políticos de los minipúblicos. En particular, muestro cómo la defensa de los usos de los minipúblicos basados en la "confianza" ciega es altamente problemática y abogo, en cambio, por los usos participativos de los minipúblicos que realmente empoderarían a la ciudadanía.

\section{Populismo y representación encarnada}

De acuerdo con el Oxford English Dictionary, el populismo "intenta apelar a la gente común que siente que sus preocupaciones son ignoradas por las élites establecidas." La apelación del populismo a la gente común parece tener su origen en una preocupación por la inclusión. Sin embargo, también conlleva una parcialidad excluyente, en tanto que aspira a definir quién pertenece al "pueblo" y quién no. Como bien indica Urbinati en su Teoría política del populismo,

si bien la concepción populista del pueblo pone el acento en la inclusión de la gente "común," no podemos dejar de advertir que este proceso de inclusión es posible gracias a un proceso paralelo de exclusión: el establishment político es la externalidad básica contra la cual el populismo sitúa al pueblo y sin la cual el populismo no puede existir... El populismo está estructuralmente atravesado por una parcialidad radical en su interpretación del pueblo y de la mayoría. (2019, p. 2).

La parcialidad excluyente del populismo tiene su origen en otro de sus aspectos característicos, a saber, la presencia de un líder fuerte que supuestamente encarna al pueblo y que puede, por ello, articular sus demandas contra las élites políticas y otros "enemigos del pueblo". A diferencia de la representación electoral, aquí el líder no representa al pueblo porque el pueblo le haya transferido autoridad política alguna. Al contrario, según el populismo, el líder encarna al pueblo y es en virtud de esta relación directa y sin mediaciones, que puede decirse que lo representa.

Yves Sintomer ilustra la idea de la "representación encarnada" con algunos ejemplos útiles:

Las palabras "representación encarnada" aparecen de forma explícita en gran parte de la lógica cesarista, ya sea populista o neopopulista. Tal como escribió un teórico del Segundo Imperio en 1853 "El emperador no es un hombre, es un pueblo," algo que parece hacerse eco en aquella proclamación del ex presidente venezolano Hugo Chávez: "Exijo lealtad absoluta a mi liderazgo porque no soy yo mismo ... no soy un individuo, soy el pueblo"; una proclama que replicaron dos años después sus partidarios cuando, reunidos masivamente con motivo de su funeral, retomaron un lema del régimen: “¡Todos somos Chávez!.” (2013, p. XVIII).

Solo porque (y en la medida en que) el líder es "uno de ellos" puede pretender representarlos. Y es precisamente esta relación directa entre el líder y su pueblo la que hace "sospechosos" a actores intermediarios como los partidos, los medios de comunicación independientes, las normas institucionales, la burocracia, los organismos de control, etc. Obviamente, la relación de "encarnación" solo puede funcionar bajo un supuesto de homogeneidad. El líder sólo puede ser "como el pueblo" si el pueblo mismo es un cuerpo homogéneo y unificado capaz de tener una relación de identidad (o al menos de suficiente similitud) con él, solo así es posible justificar la afirmación de encarnación. Si el pueblo estuviera dividido en grupos plurales o dispares

gran medida a la lente de la democracia participativa, ha pasado desapercibido el hecho de que, con respecto a la mayoría de los ciudadanos, las funciones de estas instituciones participativas son menos participativas que representativas (cf. Stephan 2004; Brown 2006). De hecho, la mayoría de las instituciones participativas están diseñadas de tal manera que algunos ciudadanos representan a otros, ya sea directamente, o -más a menudo- a través de la pretensión de representar las perspectivas, las opiniones, los bienes, los intereses y los valores de aquellos que no están presentes en el proceso (Fishkin 1995; Hanley et al. 2001; Lenaghan 1999; Parkinson 2003; Smith y Wales 2000; Ward et al. 2003). Dado que la mayoría de los ciudadanos no participan a través de estas instituciones - sujetas, como están, a las mismas limitaciones de escala y complejidad que otras instituciones- deberíamos concebirlas como órganos representativos" (2008, pp. 56-57).

3* N. de T.: Traducimos embodiment por encarnación, siguiendo la idea expuesta más abajo de un líder que encarna al pueblo. 
con puntos de vista, valores, intereses y objetivos políticos en conflicto, el líder no podría ser simultáneamente como todos ellos. Al ser como algunos de ellos, necesariamente sería distinto de otros. La literatura sobre el populismo suele señalar que este elemento de homogeneidad no es más que una construcción ficticia del supuesto "pueblo real" por parte de los propios líderes populistas - una construcción que a su vez justifica la exclusión política de las minorías opositoras transformándolas en "enemigas del pueblo"- $-{ }^{4}$ Sea como fuere, lo que importa en nuestro contexto es advertir que el supuesto de homogeneidad y la consiguiente exclusión de las minorías heterogéneas son componentes necesarios de la idea de "representación encarnada."

\section{Minipúblicos y representación encarnada}

Dentro de la teoría democrática contemporánea está surgiendo una concepción de la democracia que no está directamente relacionada con el populismo, pero que también se basa en la idea de "representación encarnada." Para simplificar, la llamaré concepción "lotocrática" de la democracia. Muchas de las personas que defienden esta concepción se ven a sí mismas como demócratas radicales. Sostienen que la inclusión política y la equidad se lograrían mejor si las democracias incorporaran procedimientos de sorteo -como la lotería o la selección aleatoria entre la gente común- en lugar de elegir a los representantes de las élites políticas. Por esta razón, depositan sus esperanzas democráticas en el uso generalizado de jurados de ciudadanos, asambleas de ciudadanos, encuestas deliberativas, etc. ${ }^{5}$ Muchos de los defensores de este enfoque recomiendan con entusiasmo que se les otorgue un estatus consultivo o incluso decisorio a estas instituciones innovadoras. Las propuestas más ambiciosas pretenden complementar o, incluso, sustituir parcialmente a las asambleas legislativas de representantes electos por asambleas de ciudadanos seleccionados al azar. Dado que el objetivo de los lotócratas es aumentar la participación de los ciudadanos comunes en la toma de decisiones políticas, la dependencia populista de un líder está totalmente ausente de este modelo. Pero, al igual que los populistas, los lotócratas suelen albergar una profunda sospecha hacia las élites políticas. En este sentido, la definición de populismo del Oxford English Dictionary se ajusta también al modelo lotocrático, que pretende "apelar a la gente común que siente que sus preocupaciones son ignoradas por las élites establecidas."

Sin embargo, para los fines de este trabajo, también es importante destacar algunas diferencias clave entre el modelo lotocrático y el modelo populista de la "representación encarnada." Desde un punto de vista puramente formal, la principal diferencia es que en el modelo de representación populista, en donde el líder encarna al pueblo, se trata de una relación de uno a muchos; mientras que en el modelo lotocrático, en donde una selección aleatoria de ciudadanos comunes "reflejan al pueblo," se trata de una relación de muchos a muchos. Esta diferencia formal tiene consecuencias importantes. Para comenzar, mientras que la afirmación populista de que el o la líder encarna al pueblo porque es "como él" puede ser impugnada, y a menudo lo es, por aquellas personas que no se ven reflejadas en el o la líder, es más difícil desafiar la afirmación de que una selección aleatoria de ciudadanos comunes encarna al pueblo porque sus miembros son "como ellos." Este es el caso especialmente cuando se utilizan técnicas de estratificación en la selección aleatoria con la intención explícita de generar un grupo que "refleje al pueblo." En suma, me preocupa que los rasgos problemáticos de la noción de "representación encarnada" puedan ser operativos dentro de las concepciones lotocráticas, pero mucho más difíciles de detectar que en las concepciones populistas. Ningún líder, partido político u organización puede equiparar la similitud entre una selección aleatoria y estratificada de ciudadanos comunes y la ciudadanía que reflejan. Precisamente porque los grupos de ciudadanos que participan en las selecciones aleatorias y estratificadas se parecen tanto a la ciudadanía que reflejan, albergan la posibilidad de convertirse en los "usurpadores definitivos."

En lo que sigue, exploro esta preocupación a través de un análisis minucioso de los rasgos específicos de la noción de "representación encarnada" que subyace a las concepciones lotocráticas de la democracia y sus consecuencias negativas potenciales. Aunque la noción de "ciudadano-representante" es esencial en este modelo, todavía no se ha explorado en profundidad. ${ }^{6}$ Por ejemplo, no está claro cómo un ciudadano puede desempeñar simultáneamente el papel de ciudadano y de representante con respecto a una misma decisión política. Tampoco está claro qué obligaciones tienen los ciudadanos-representantes hacia el resto de la ciudadanía que no participa en el proceso de toma de decisiones: ¿pueden los ciudadanos-representantes simultáneamente formar parte del poder "constituyente" y del poder "constituido"? ¿Pueden rendir cuentas de manera significativa a los que no participan $y$, al mismo tiempo, seguir ejerciendo sus derechos como ciudadanos capaces de tomar sus propias decisiones libremente? Mi análisis no pretende responder a estas intrincadas preguntas. Las menciono solo para indicar cómo, en ausencia de tales respuestas, no podemos evaluar la legitimidad democrática de las propuestas lotocráticas. El modesto objetivo de mi análisis aquí

\footnotetext{
Ver, por ejemplo, Jan-Werner Müller (2016).

Para un análisis detallado de las principales similitudes y diferencias entre estos tipos de minipúblicos, véase Smith y Setälä (2019). Para más información sobre los jurados de ciudadanos, véase Crosby y Nethercut (2005); sobre las asambleas de ciudadanos véase Fournier, van der Kolk y Carty (2011) y Warren \& Pearse (2008), y sobre las encuestas deliberativas véase Fishkin (2018).

Para algunos trabajos interesantes sobre esta temática, véase Brown (2006), Warren (2008), Landemore (2020).
} 
es desafiar el supuesto de que la noción de "representación encarnada" puede aplicarse de forma fructífera a las instituciones lotocráticas, resaltando con ello algunas de las consecuencias preocupantes del modelo lotocrático.

\section{Ciudadanos-representantes: ¿los usurpadores definitivos?}

Los lotócratas defienden la proliferación de minipúblicos y otras instituciones lotocráticas como formas de democratización radical. ${ }^{7}$ Sus propuestas de otorgar un estatus decisorio a las asambleas legislativas compuestas por sorteo, en lugar de por elección, se basan en la idea de la representación como “encarnación." Los ciudadanos deberían confiar en las decisiones recomendadas por dichas instituciones porque los participantes son como ellos ${ }^{8}$ Como los participantes son ciudadanos comunes, pueden hablar como el pueblo y no simplemente en su nombre. En otras palabras, los minipúblicos deben tener autoridad consultiva o incluso decisoria no porque la ciudadanía haya autorizado o seleccionado a sus miembros a través de los mecanismos habituales de representación electoral. Más bien, sería simplemente porque ellos son un "reflejo del pueblo." De hecho, las técnicas para la selección aleatoria y estratificada parecen respaldar la afirmación de que los ciudadanos comunes que participan en los minipúblicos son en realidad un reflejo exacto de la población en su conjunto; en consecuencia, sus opiniones, intereses, valores, etc., reflejan los de la población en general. Más aún, las innovaciones institucionales que recrean un microcosmos del pueblo pueden aportarnos información vital. Así como los juicios iniciales de los participantes reflejan la opinión pública cruda y (habitualmente) desinformada que puede captarse en los sondeos habituales, es plausible suponer que los juicios de los participantes tras la experiencia deliberativa reflejan lo que la gente pensaría si estuviera informada y tuviera la oportunidad de deliberar sobre el asunto. En este sentido, representan "los juicios ponderados [considered judgements] del pueblo" (Fishkin, 2009, p. 28). ${ }^{9}$

Este enfoque se suele asociar con un modelo de representación por selección en contraste con un modelo de representación por sanción. ${ }^{10}$ Mientras que un modelo de sanción espera que los representantes sigan con exactitud las opiniones de sus electores so pena de perder su cargo, un modelo de selección espera que los electores elijan a representantes con intereses, valores y objetivos que estén en gran medida alineados con los suyos, de modo que los representantes posean razones exógenas y automotivadas para implementar [enact] la voluntad de sus constituyentes (Miller y Stokes, 1963, citado por Mansbridge, 2009, p. 371). ${ }^{11}$ En nuestro contexto, la pregunta es si la congruencia o "coincidencia" entre los intereses y valores de los representantes y los de los ciudadanos que los eligieron, que caracteriza el modelo de selección, puede suponerse igualmente entre los intereses y valores de los integrantes del grupo seleccionado al azar y los ciudadanos no participantes que la muestra aleatoria refleja. Dado que las propuestas que confieren un estatus decisorio a los minipúblicos se justifican sobre la base de que sus participantes son como nosotros (o al menos más parecidos a nosotros que las élites políticas, los jueces, los grupos de interés u otros actores políticos), necesitamos examinar este supuesto de concordancia. El argumento involucra varias afirmaciones.

Los participantes de los minipúblicos son como nosotros en tanto que son ciudadanos comunes. Por lo que, a diferencia de los políticos, los grupos de presión y otros actores políticos, es poco probable que tengan agendas ocultas o conflictos de intereses en sus deliberaciones sobre el interés público. Podemos confiar en ellos como nuestros representantes en el sentido de que no necesitamos vigilarlos ni amenazarlos con sanciones porque están motivados independientemente a averiguar lo que es mejor para la comunidad política. Ahora bien, siguiendo el modelo de representación por selección, se afirma que los participantes de los minipúblicos son como nosotros en un sentido más fuerte: supuestamente comparten nuestros intereses, valores, objetivos políticos, etc. ${ }^{12}$ Por eso se supone que debemos confiar en ellos, no solo en el sentido de que no necesitamos amenazarlos con sanciones para que rindan cuentas, sino también, y en un sentido más fuerte, porque podemos suponer que sus recomendaciones coinciden con lo que nosotros habríamos pensado si hubiéramos participado. En resumen, debemos confiar en ellos en el sentido fuerte de aceptar sus recomendaciones como si fueran nuestras (por ejemplo, a la hora de votar en referendos). ${ }^{13}$

Véase, por ejemplo, Sintomer (2018).

Véase Sintomer (2013).

En esta sección y la siguiente tomo elementos de Lafont (2020).

10 Para un análisis en profundidad del contraste entre el modelo de selección y el de sanción de la representación, véase Mansbridge (2009). Para el contraste entre los modelos de selección y sanción en relación con los minipúblicos como encuestas deliberativas, véase Mansbridge (2010). Para un interesante análisis del contraste entre estos dos modelos de representación bajo las rúbricas "receptivo" e "indicativo," véase Pettit (2010).

11 Es importante tener en cuenta que tanto el modelo de selección como el de sanción son modelos electorales de representación, mientras que en el caso de los minipúblicos no hay elección alguna, es decir, los ciudadanos no seleccionan a ninguno de los participantes. Las consecuencias de esta cuestión se analizan en la última sección del artículo.

12 Como indica Mansbridge (2009) con respecto al alineamiento de objetivos entre el agente y el director bajo el modelo de selección, "el alineamiento de objetivos puede tener lugar no sólo respecto a la comprensión de lo que es mejor para la nación en su conjunto, sino también respecto a lo que es mejor para individuos o comunidades particulares como los agricultores, los mineros o los residentes de los centros urbanos" (p. 380).

13 Para una defensa de estos usos de los minipúblicos basados en la confianza, véase MacKenzie y Warren (2012) y Warren y Gastil (2015). 
Ahora bien, mientras que la primera afirmación es plausible, la segunda es problemática. Dado que en las sociedades pluralistas hay tanto desacuerdo sobre cuestiones políticas y morales entre los ciudadanos, la afirmación más fuerte difícilmente puede ser cierta de una muestra genuinamente representativa de la población. Cuanto más se incluyan en la muestra diversas perspectivas evaluativas (relativas a interpretaciones de necesidades, valoraciones, puntos de vista globales, etc.), menos sentido tendrá para los ciudadanos no participantes suponer que sus propios intereses, valores y objetivos políticos coincidirán invariablemente con los de la mayoría del grupo aleatorio independientemente del tema cuestión. Los no participantes no pueden dar por sentado que las recomendaciones de la mayoría del minipúblico reflejan lo que ellos habrían pensado si hubieran participado. Pues, en principio, lo contrario es igualmente posible. A fin de cuentas, los participantes que están en desacuerdo con la mayoría han revisado la misma información y han deliberado tanto como los demás y, sin embargo, han llegado a la conclusión contraria. Incluso si los ciudadanos pudieran confiar en que todos los participantes estarán genuinamente interesados en averiguar qué es lo mejor para la comunidad, saben que en las democracias pluralistas hay disputas en curso [ongoing contestation] entre diversos puntos de vista y valores sociales, morales, éticos, religiosos y económicos, y que ello influye significativamente en las consideraciones políticas y en los objetivos de las políticas públicas. Lejos de constituir un cuerpo homogéneo, los ciudadanos en las sociedades pluralistas comprometidos con el mantenimiento de instituciones libres tienen muchos desacuerdos políticos con sus conciudadanos.

Por ello, en sociedades pluralistas, el modelo de selección de la representación parece plausible a menor escala. Los ciudadanos pueden confiar en aquellos partidos políticos, organizaciones de la sociedad civil o individuos que compartan sus intereses, valores y objetivos políticos. Pero por esta misma razón no tendría sentido también confiar en los partidos políticos, organizaciones e individuos que defienden los puntos de vista, valores y objetivos políticos contrarios, sean los que sean. Si confío en las recomendaciones de Oxfam sobre cómo luchar contra la pobreza, no puedo confiar también en las recomendaciones contrarias de, por ejemplo, la Cámara de Comercio. Si confío en las recomendaciones de Planned Parenthood sobre la salud reproductiva de las mujeres, no puedo confiar al mismo tiempo en las recomendaciones contrarias de, por ejemplo, la Liga de Acción ProVida. Dado que no puedo confiar simultáneamente en los puntos de vista, valores y objetivos políticos en conflicto de esta multiplicidad de actores, tampoco puedo confiar en las recomendaciones de la mayoría de la muestra sin saber primero si se han posicionado del lado del espectro político que yo habría adoptado si hubiera participado.

Por supuesto, si los contenidos y las deliberaciones se hacen públicos, los ciudadanos siempre podrán averiguar si ese es el caso. Pero una vez que lo hagan, dejarán de confiar en el minipúblico. Confiarán en sí mismos. Y lo que es más importante, muchos de ellos descubrirán que la mayoría de la selección no es como ellos, ya que en realidad se oponen a sus opiniones, valores y objetivos políticos respecto al tema en cuestión. En este punto, la afirmación de que los no participantes deberían confiar en las recomendaciones de los minipúblicos porque los participantes son como ellos se derrumba inevitablemente. Porque el hecho de que la muestra aleatoria sea un microcosmos del pueblo considerado colectivamente significa que, en las cuestiones controvertidas, habrá una mayoría que defienda una opinión y una minoría que defienda la opinión opuesta. Esto significa que no puede ser cierto de todas las personas consideradas individualmente que la mayoría de la muestra sea como ellas. Pero si esto es así, ¿en qué sentido podemos decir que los participantes en la muestra son sus representantes? Si la mayoría de la muestra no es como ellos ni les rinde cuentas, entonces, ¿cuál es la justificación para esperar que los ciudadanos no participantes simplemente confíen ciegamente en esta mayoría? Dado que los ciudadanos no han seleccionado a sus representantes para participar en el minipúblico, ninguno de ellos tiene una razón particular para suponer que las recomendaciones de la mayoría o las de la minoría son las que coinciden con lo que ellos habrían pensado si se hubieran informado y reflexionado por su cuenta.

A diferencia del modelo estándar de selección de la representación en el que los ciudadanos eligen a sus representantes en función de sus propios intereses, valores y objetivos políticos, cuando se trata de minipúblicos quizá el argumento no sea que los ciudadanos deben confiar en la mayoría porque es como ellos, sino que deben confiar en ella porque es como la mayoría del pueblo. ${ }^{14}$ Pero, ¿lo es?

\section{La ilusión de la democracia o “¡Cuidado con los usurpadores!”}

Antes de la experiencia deliberativa, es trivialmente verdadero afirmar que quienes participan en el minipúblico son como el pueblo en el sentido de que las perspectivas de los ciudadanos de la muestra aleatoria reflejan con

\footnotetext{
No se me ocurre ninguna interpretación del modelo de selección de la representación que haría plausible afirmar que los ciudadanos deberían confiar en que la opinión ponderada de la mayoría de un grupo aleatorio de personas coincidirá invariablemente con lo que habrían pensado ellos si hubieran sido informados. Analizo las dificultades de esta afirmación en Lafont (2015, p. 54-57). Pero, independientemente de que esta visión de la representación pueda considerarse plausible, el problema en nuestro contexto es que la afirmación "modificada" del reflejo entre ambos grupos, en la que se basa, es falsa. En la etapa postdeliberativa empoderada [post-deliberative empowered stage], es frecuente que la mayoría de la muestra aleatoria ya no refleje la opinión de la mayoría del pueblo.
} 
exactitud las opiniones del conjunto de la población. Es por esto que las encuestas regulares pueden usarse (de forma más o menos fiable) para averiguar las opiniones de la población, a pesar de que de hecho solo se entrevista a un puñado de ciudadanos seleccionados al azar. Sin embargo, una vez que se implementa el filtro deliberativo -que es el objetivo principal para el que se organizan minipúblicos deliberativos- las opiniones de los participantes sufren transformaciones significativas y, a veces, drásticas. Pero, precisamente por eso, pretender que la voz de los que participan en los minipúblicos después de la deliberación sea la voz del pueblo sería un claro caso de usurpación, sobre todo en aquellos casos en los que consta explícitamente que disienten del pueblo real. La tentación populista de "hablar por el pueblo" es común entre actores políticos de todo tipo, pero la flagrante disimilitud entre estos actores y la población real contribuye a socavar tales pretensiones. Por el contrario, la similitud entre los participantes de los minipúblicos y el pueblo en la etapa inicial, predeliberativa, hace que su disimilitud después de la deliberación, en la etapa empoderada, sea más difícil de detectar. Como indiqué antes, por esa razón podrían convertirse en los "máximos usurpadores." Veamos por qué.

Los participantes de los minipúblicos se supone que son representativos de la ciudadanía en general en el sentido descriptivo del término. Desgraciadamente, esto solo es cierto (si es que lo es) con respecto a las opiniones "crudas" que tienen antes del proceso de deliberación, ya que el objetivo de pasar por ese proceso es justamente provocar una transformación de sus juicios iniciales, la cual da lugar a juicios cualitativamente diferentes que, por esta misma razón, ya no son representativos de las perspectivas actuales de la ciudadanía. Sea como fuere, lo que está claro es que los participantes seleccionados no actúan como representantes de los grupos a los que representan en un sentido descriptivo. No se supone en absoluto que las participantes femeninas han de defender las opiniones de las mujeres ni los californianos las opiniones de otros californianos. Participan como ciudadanos individuales con total libertad para expresar sus perspectivas y opiniones y para cambiarlos de la manera que consideren oportuno. ${ }^{15}$ Pero, por esa misma razón, no le rinden cuentas a los ciudadanos que no participan en el minipúblico. La razón se debe a la peculiar naturaleza de la representación inherente al procedimiento de muestreo estratificado aleatorio.

Dado que se supone que la muestra aleatoriamente seleccionada "refleja a la población" en su conjunto, sus miembros se consideran indicadores fiables de los juicios de la ciudadanía (por ejemplo, de lo que la ciudadanía pensaría sobre un asunto político particular si tuviera la oportunidad de informarse y deliberar en buenas condiciones). Pero precisamente por esa razón no hay espacio para que los no participantes exijan a los participantes de los minipúblicos que les rindan cuentas por las recomendaciones específicas que hacen, del mismo modo que no se puede exigir a un termostato que rinda cuentas por la temperatura específica que fiablemente indica.

Además, puesto que lo que las recomendaciones de los minipúblicos se supone que reflejan no son los juicios actuales de ningún ciudadano o grupo en particular, sino los juicios considerados del pueblo en su conjunto, el hecho de que un número cualquiera de no participantes no esté de acuerdo con esas recomendaciones no ofrece ninguna base para cuestionar la fiabilidad de los participantes en los minipúblicos. Después de todo, no se les selecciona para que indiquen de modo fidedigno la opinión pública actual, sino la contrafáctica, es decir, lo que la gente pensaría si hubiera reflexionado sobre el asunto en buenas condiciones epistémicas. En caso de rendir cuentas a alguien, sería a la ciudadanía contrafáctica cuyos juicios ponderados se supone que fiablemente indican, y no los juicios prerreflexivos de la ciudadanía real. Dado esto, ¿por qué debería considerar la ciudadanía que los juicios de estos participantes tienen más fuerza de recomendación que sus propias opiniones?

\section{¿Son la mayoría de mis conciudadanos “como yo"? El problema de la deferencia ciega}

En las sociedades pluralistas, es probable que los participantes de los minipúblicos discrepen sobre cuestiones políticas controvertidas. Esto es particularmente evidente en casos como las encuestas deliberativas, en las que los participantes no están obligados a llegar a un consenso. El hecho de que una muestra aleatoria sea un microcosmos de la población considerada colectivamente significa que, para cuestiones controvertidas, habrá una mayoría defendiendo una opinión y una minoría defendiendo la opinión opuesta. Por consiguiente, no

\footnotetext{
Brown (2006) resume algunas de las dificultades que conlleva la aplicación de la noción de representación descriptiva a los minipúblicos o paneles de ciudadanos: "Primero, cualquier miembro del panel en particular pertenecerá a múltiples categorías estadísticas, y es imposible saber de antemano cómo los individuos concretos priorizan sus diversas identidades en su concepción de sí mismos y en su comportamiento. Segundo, siempre es posible encontrar personas que se ven a sí mismas como miembros de un grupo social pero que carecen de al menos algunos de los atributos supuestamente definitorios del grupo, y viceversa. Tercero, seleccionar a los panelistas como representantes de los intereses del grupo sobre la base de su identidad social sugiere falsamente que las personas solo son capaces de representar los intereses de su propio grupo social. Cuarto, incluso las personas que se definen a sí mismas como miembros de un determinado grupo social pueden diferir mucho en sus valores e intereses políticos. Y finalmente, dar por supuesto que los participantes tienen intereses fijos asociados a categorías sociales particulares obtura el proceso de adquisición de información y transformación de intereses, que es lo que la deliberación pretende fomentar. En resumen, no todos los grupos identitarios son grupos de interés, y la identidad del grupo a menudo existe antes de cualquier sentido de interés compartido, y entra en conflicto con él” (p. 218).
} 
puede ser cierto de todas las personas consideradas individualmente que la mayoría de la muestra aleatoria sea como ellas. En ausencia de información sobre las razones, valores, intereses, etc. específicos que subyacen a la recomendación de la mayoría, ningún ciudadano tiene razón alguna para suponer que la recomendación de la mayoría o la de la minoría coincide con lo que ellos mismos habrían pensado si hubieran deliberado. De hecho, precisamente porque los minipúblicos son un microcosmos del pueblo en su conjunto, no es posible que todos sean como yo. Algunos compartirán mis intereses y valores y otros no. Dado que no hay ninguna razón en particular para suponer que las recomendaciones de los minipúblicos coincidirán siempre con las que aprueban los participantes que compartan mis intereses y valores (ya sea antes o después de la deliberación), no tengo más razones para aceptar las recomendaciones o decisiones de la mayoría de los minipúblicos, de las que tendría para aceptar las de la mayoría de mis conciudadanos en general. La razón es simple: el mero hecho de que una decisión política cuente con el apoyo de la mayoría no añade nada a las razones en su favor. Claramente, pedir a quienes no están de acuerdo con una determinada recomendación que defieran ciegamente a la mayoría del minipúblico por el mero hecho de que han considerado otras razones y han llegado a una conclusión diferente sería sencillamente injustificado. Pero esto también revela qué tiene de malo esperar lo mismo de los votantes indecisos o desinformados.

Al esperar que los ciudadanos defieran ciegamente a las decisiones políticas de un grupo de ciudadanos seleccionados al azar, el uso generalizado de los minipúblicos para la toma de decisiones disminuirá en lugar de aumentar la capacidad de los ciudadanos de apropiarse e identificarse con las leyes y políticas a las que están sujetos, tal y como requiere el ideal democrático de autogobierno. De hecho, el efecto a largo plazo de tomar decisiones políticas basadas en los juicios deliberados de los participantes de los minipúblicos en lugar de los juicios reales de la ciudadanía es que estos últimos tendrán dificultades para verse a sí mismos como participantes en un proyecto democrático de autogobierno. Institucionalizar minipúblicos con la esperanza de que estos piensen y decidan por el resto de la ciudadanía no sería una forma de aumentar el control democrático ciudadano sobre el proceso político. Desde el punto de vista democrático, la diferencia fundamental radica en si los minipúblicos se conciben como maneras de empoderar a la ciudadanía o de pasarla por alto.

Para que los minipúblicos puedan tener un efecto democratizador, hay que pensar en formas de utilizarlos para mejorar la participación y la influencia de la ciudadanía en su conjunto en el proceso político.

Desde esa perspectiva, los minipúblicos podrían cumplir dos importantes funciones democráticas: primero, podrían contribuir a mejorar la calidad de la deliberación pública, de modo que la ciudadanía también pueda alcanzar una opinión ponderada sobre las decisiones políticas importantes a las que está obligada a obedecer y, segundo, podrían contribuir a hacer el sistema político más receptivo a la opinión pública ponderada sobre las decisiones políticas en cuestión. En mi libro Democracia sin atajos ${ }^{16}$ ofrezco un análisis detallado de varios modos en que los minipúblicos podrían desempeñar estas importantes funciones. No tengo espacio para reproducir ese análisis aquí. Pero lo importante en este contexto es que los usos de los minipúblicos que tienen como objetivo informar y empoderar a la ciudadanía no se basan en una comprensión de los participantes de los minipúblicos como sus "representantes" políticos, es decir, como miembros de un órgano representativo con la autoridad para hablar y actuar en su nombre porque supuestamente encarnan al "pueblo."

\section{Una alternativa a la usurpación: De ser como nosotros a hablar con nosotros}

Los ciudadanos pueden tener muy buenas razones para tomarse en serio las opiniones y recomendaciones de los participantes de los minipúblicos en la medida en que son indicativas de la variedad de puntos de vista políticos ponderados con los que sus conciudadanos podrían identificarse. Para ser útiles en este sentido, los minipúblicos tendrían que dar a conocer no solo una recomendación o decisión, sino también la información que consideran esencial para una correcta comprensión de lo que está en juego en la decisión en cuestión, así como las razones y los argumentos más importantes a favor y en contra de la recomendación que ha logrado el apoyo mayoritario. La ciudadanía podría utilizar esta valiosa información para decidir sobre los temas que se debaten. No obstante, los ciudadanos no participantes no tienen ninguna razón para suponer una mayor congruencia entre sus intereses y valores y los de la mayoría de los participantes del minipúblico, que entre los intereses y valores de los de la minoría para cualquier cuestión. Por este motivo, no tienen ninguna buena razón para confiar en la recomendación de la mayoría y seguirla ciegamente.

Los ciudadanos pueden confiar en la información relevante seleccionada colectivamente por los minipúblicos. También pueden confiar en que las razones escogidas colectivamente a favor y en contra de las decisiones de la mayoría reflejan con exactitud las razones que con mayor probabilidad resonarán en la ciudadanía. Pero para determinar si apoyar o no la recomendación de la mayoría tienen que averiguar por sí mismos cuáles de estas razones contrapuestas son más congruentes con sus propios intereses, valores y objetivos políticos. Esto no debería ser sorprendente si tenemos en cuenta que la relación de representación entre la ciudadanía en su totalidad y la muestra aleatoria es solo una relación de muchos a muchos. Dado que la

En Lafont (2020) ofrezco un análisis detallado de los posibles usos políticos de los minipúblicos para cumplir ambas funciones. 
muestra en su conjunto representa al conjunto de la ciudadanía, parece plausible suponer que la primera puede proporcionar información interesante sobre la segunda a los ciudadanos no participantes. Sin embargo, esto es así únicamente respecto de la información que ha sido seleccionada colectivamente por todos los miembros de la muestra aleatoria y, por lo tanto, es representativa de la muestra tomada en su totalidad. Por el contrario, dado que los ciudadanos no participantes no han escogido a ningún miembro concreto de la muestra, no hay una relación de representación individual entre ellos y un miembro concreto o subconjunto específico de ese grupo (no hay una relación de representación de uno a uno, ni de uno a muchos). Por eso no tienen razón alguna para esperar una mayor congruencia entre sus intereses y valores y los de algún subconjunto concreto de la muestra aleatoria, y por eso no tienen ninguna buena razón para confiar en una recomendación que haya sido seleccionada solo por un subconjunto de la muestra, independiente de lo grande o pequeño que sea cada subconjunto.

Por lo tanto, el problema de afirmar que los ciudadanos no participantes deben confiar en la recomendación de la mayoría no es que se apoye en el supuesto de que la muestra aleatoria es un reflejo de "el pueblo." Al contrario. Precisamente si el supuesto del reflejo es cierto, no es posible que todos los ciudadanos sean como la mayoría y no como la minoría de la muestra. Por tanto, esa afirmación no puede justificar una recomendación generalizada de confiar en la mayoría de la selección. Incluso si uno supone que la selección refleja al pueblo en su conjunto, esto no dice nada sobre qué subconjunto de la muestra es más congruente con los intereses y valores de cada ciudadano no participante. De hecho, para suponer que la mayoría de la muestra representa invariablemente al "pueblo" (y, por tanto, que sus recomendaciones deberían ser seguidas ciegamente por todos los ciudadanos no participantes) uno necesitaría adoptar el mayoritarismo excluyente que caracteriza al populismo y suponer que los intereses y valores de la minoría de participantes no son congruentes con los del "pueblo real" y que, por tanto, deberían ser simplemente rechazados o ignorados por los ciudadanos no participantes.

Como mínimo, esto indica que para conceptualizar adecuadamente la relación entre los participantes y los no participantes en minipúblicos no son útiles ni el modelo de selección ni el de encarnación del pueblo. Los participantes de los minipúblicos no pueden hablar por nosotros porque no los hemos elegido para hacerlo. Y no pueden hablar como nosotros porque nosotros, el pueblo, no somos un grupo políticamente homogéneo. En lugar de ello, deberían hablarnos a nosotros, sus conciudadanos, para que podamos mejorar de forma colectiva la calidad deliberativa de nuestras decisiones políticas.

\section{Referencias bibliográficas}

Brown, Mark B. (2006). Survey article: Citizen's panels and the concept of representation [Artículo de encuesta: Los paneles de ciudadanos y el concepto de representación], The Journal of Political Philosophy, 14(2), 203-225.

Crosby, Ned y Nethercut, Doug (2005). Citizen juries: Creating a trustworthy voice of the people [Jurados populares: creando una voz de la gente digna de confianza]. En J. Gastil y P. Levine (Eds.), The deliberative democracy handbook (pp. 111-119), Jossey-Bass. doi: https://doi.org/10.1007/s11077-005-0870-3

Fishkin, James S. (2009). When the people speak: Deliberative democracy and public consultation [Cuando la gente habla: democracia deliberativa y consulta pública]. Oxford University.

Fishkin, James S. (2018). Democracy when the people are thinking [La Democracia cuando la gente piensa]. Oxford University.

Fournier, Patrick, van der Kolk, Henk y Carty, R. Kenneth (2011). When citizens decide: Lessons from citizen assemblies on electoral reform [Cuando los ciudadanos deciden: lecciones de las asambleas ciudadanas sobre la reforma electoral]. Oxford University.

Lafont, Cristina (2015). Deliberation, participation and democratic legitimacy: Should deliberative minipublics shape public policy? [Deliberación, participación y legitimidad democrática: ¿Deberían los minipúblicos deliberativos dar forma a las políticas públicas?]. Journal of Political Philosophy, 23(1), 40-63. doi: https://doi.org/10.1111/jopp.12031

Lafont, Cristina (2020). Democracy without shortcuts. A participatory conception of deliberative democracy [Democracia sin atajos. Una concepción participativa de la democracia deliberativa]. Oxford University.

Landemore, Hélène (2020). Open democracy. Reinventing popular rule for the 21st century [Democracia abierta. Reinventar el dominio popular para el siglo XXI]. Princeton University.

MacKenzie, Michael. K. y Warren, Mark E. (2012). Two trust-based uses of minipublics in democratic systems [Dos usos de los minipúblicos basados en la confianza en los sistemas democráticos]. En J. Parkinson, y J. Mansbridge (Eds.). Deliberative systems. Deliberative democracy at the large scale (pp. 95-124). Cambridge University.

Mansbridge, Jean (2010). Deliberative polling as the gold standard [El sondeo deliberativo como estándar de oro]. The Good Society, 19(1), 55-62. doi: https://doi.org/10.5325/goodsociety.19.1.0055.

Mansbridge, Jean (2009). A "selection model" of political representation [Un "modelo de selección" de representación política]. Journal of Political Philosophy, 17(4), 369-98. doi: https://doi.org/10.1111/j.1467-9760.2009.00337

Müller, Jan-Werner. (2016). What is populism? [¿Qué es el populismo?]. University of Pennsylvania. 
Pettit, Philip (2010). Representation, responsive and indicative [Representación, receptiva e indicativa.]. Constellations, $17(3), 426-434$

Sintomer, Yves (2013). The meanings of political representation: Uses and misuses of a notion [Los significados de la representación política: usos y usos indebidos de la noción]. Raisons Politiques, 50, 13-34. doi: https://doi.org/10.3917/ rai.050.0013

Sintomer, Yves (2018). From deliberative to radical democracy? Sortition and politics in the twenty-first century [¿De la democracia deliberativa a la radical? Sorteo y política en el siglo XXI]. Politics \& Society, 46(3), 337-357. doi: https:// doi.org/10.1177/0032329218789888

Smith, Graham, y Setälä, Maija (2019). Mini-publics and deliberative democracy [Minipúblicos y democracia deliberativa]. En A. Bächtiger, J. S. Dryzek, J. Mansbridge, y M. Warren (Eds.), The Oxford handbook of deliberative democracy (pp. 300-314). Oxford University.

Urbinati, Nadia (2019). Political theory of populism [La teoría política del populismo]. Annual Review of Political Science, 22, 111-127. doi: https://doi.org/10.1146/annurev-polisci-050317-070753

Warren, Mark E. (2008). Citizen representatives [Representantes ciudadanos]. En M. Warren y H. Pearse (Eds.). Designing deliberative democracy. The British Columbia citizens assembly (pp. 50-69). Cambridge University.

Warren, Mark E. y Gastil, John (2015). Can deliberative minipublics address the cognitive challenges of democratic citizenship? [¿Pueden los minipúblicos deliberativos abordar los desafíos cognitivos de la ciudadanía democrática?]. The Journal of Politics, 77(2), 562-574. doi: https://doi.org/10.1086/680078

Warren, Mark E. y Pearse, Hilary (Eds.). (2008). Designing deliberative democracy. The British Columbia citizens assembly [Diseñando democracia deliberativa. La asamblea de ciudadanos de Columbia Británica]. Cambridge University. doi: https://doi.org/10.1017/CBO9780511491177 